\title{
Results of ECOG-ACRIN E2108 Trial: Is This the End of Primary Surgery in Metastatic Breast Cancer?
}

\author{
Paulo Luz
}

Department of Medical Oncology, Centro Hospitalar Universitário do Algarve; CBIOS - Research Center for Biosciences and Health Technologies, Universidade Lusófona de Humanidades e Tecnologias, Faro, Portugal

Cite this article as: Luz P. Results of ECOG-ACRIN E2108 Trial: Is This the End of Primary Surgery in Metastatic Breast Cancer?. Eur J Breast Health 2021; 17(1): 84-85.

\section{Dear Editor,}

We were looking forward to the results of the ECOG-ACRIN E2108 trial presented at ASCO 2020 in order to fully understand the role of primary tumor surgery in patients with stage IV breast cancer, as some studies presented with conflicting data (1-3).

In this trial, 390 patients with stage IV breast cancer and intact primary tumor who did not progress after 4-8 months of optimal systemic therapy were randomized to locoregional treatment (surgery and radiotherapy) accompanied by systemic therapy or continued systemic therapy alone. Moreover, there was no significant difference between the 3-year overall survival rate $(68.4 \%$ for the group treated with locoregional treatment vs $67.9 \%$, stratified log-rank $\mathrm{p}=0.63$, hazard ratio: $1.09,90 \%$ confidence interval: $0.80,1.49)$ and progression-free survival $(\mathrm{p}=0.40)$ between the groups. Locoregional recurrence/progression was predicted to be significantly higher in the systemic therapy alone arm (3-year survival rate $25.6 \%$ vs $10.2 \%$, Gray test $\mathrm{p}=0.003$ ). Quality of life measured by The Functional Assessment of Cancer Therapy - Breast was lower in the locoregional treatment arm than systemic therapy alone at 18 months post-randomization, but no difference was observed at 6 or 30 months.

This trial included patients who did not progress after 4-8 months of systemic therapy without differentiating the tumor burden or response (stable, partial, or complete) following treatment. In our daily practice, we only provide locoregional therapy in patients with complete positron emission tomography response but persistence of primary tumor after systemic therapy. We can ignore the psychological effects for the patient to be disease-free. This approach also makes it possible to address cessation of systemic therapy with the patient after a long disease-free period. Locoregional therapy should also be considered in cases of oligometastatic disease where local treatment for all metastatic lesions is possible, not forgetting that there is already evidence of locoregional palliation.

Badwe et al. (2) randomized de novo metastatic breast cancer for surgery first followed by systemic or non-locoregional therapy, which did not allow selecting patients according to the response to systemic therapy. In this trial, no advantages for surgery were observed in oligometastatic patients under analysis; however, it is not specified if these patients have undergone local treatment for metastatic lesions. We must also take into account that very few patients with human epidermal growth factor receptor 2 (HER2) breast cancer received anti-HER2 therapy.

Nevertheless, different findings were seen in a similar clinical trial. Soran et al. (3) also randomized patients for locoregional or systemic therapy. With a median follow up of 40 months, $41.6 \%$ of the patients survived in the locoregional group, whereas $24.4 \%$ survived in the systemic group $(\mathrm{p}=0.005)$. The proposed subgroup analysis showed that patients with luminal or HER2 disease, patients younger than 55 years, or patients with solitary bone metastasis had a significant survival benefit with initial locoregional therapy.

Much higher data are required for studying the following two scenarios: complete response of all metastatic lesions but persistence of primary tumor and in cases of oligometastatic disease with all metastatic lesions receiving local treatment. For these reasons and until more data is available on the circumstances mentioned, I believe that primary surgery for metastatic breast cancer should not be "buried" but should be considered on a case-by-case basis.

Keywords: Breast cancer, chemotherapy, radiotherapy, surgery 


\section{Luz P. Primary Surgery in Metastatic Breast Cancer}

Peer-review: Externally peer-reviewed.

Conflict of Interest: The author has no conflict of interest to declare.

Financial Disclosure: The author declared that this study received no financial support.

\section{References}

1. Khan, S, Zhao F, Solin L, Goldstein L, Cella D, Basik M, et al. A randomized phase III trial of systemic therapy plus early local therapy versus systemic therapy alone in women with de novo stage IV breast cancer: A trial of the ECOG-ACRIN Research Group (E2108). J Clin Oncol 2020; 38(18suppl): LBA2-LBA2. 10.1200/JCO.2020.38.18_ suppl.LBA2. [Crossref]

2. Badwe R, Hawaldar R, Nair N, Kaushik R, Parmar V, Siddique S, et al. Locoregional treatment versus no treatment of the primary tumour in metastatic breast cancer: an open-label randomised controlled trial. Lancet Oncol 2015; 16: 1380-1388. (PMID: 26363985) [Crossref]

3. Soran A, Ozmen V, Ozbas S, Karanlik H, Muslumanoglu M, Igci A, et al. Randomized trial comparing resection of primary tumor with no surgery in stage IV breast cancer at presentation: Protocol MF07-01. Ann Surg Oncol 2018; 25: 3141-3149. (PMID: 29777404) [Crossref] 